\title{
Influence of roundness error screw on running characteristics of sliding bearing based on SDT
}

\author{
Zhenyu Chen ${ }^{1,2}$, Wubin $\mathrm{Xu}^{1,2}$, and Bing $\mathrm{Li}^{1,2, *}$ \\ ${ }^{1}$ School of Mechanical and Traffic Engineering, Guangxi University of Science and Technology, \\ Liuzhou 545006 Guangxi, China \\ ${ }^{2}$ Guangxi Earthmoving Machinery Collaborative Innovation Center, Liuzhou 545006 Guangxi, China
}

\begin{abstract}
In order to study the influence of roundness error on the oil film characteristics of journal bearing rotor system, a dynamic model of journal bearing rotor system with roundness error was established, and a new generalized roundness error equation is derived based on the small displacement screw (SDT) theory. And the influence of roundness error screw parameter dx represented by SDT on critical speed and stability of sliding bearing is analyzed emphatically. The results show that the existence of journal roundness error is beneficial to the bearing capacity and stability of sliding bearing rotor system to a certain extent, and with the increase of roundness error screw parameter, its promoting effect is more obvious; At the same time, the critical speed of the system will increase with the increase of screw parameter, especially when eccentricity $\varepsilon>0.6$; And when Sommerfeld number $\mathrm{S}>0.6$, the roundness error of journal has little influence on stability.
\end{abstract}

Keywords: Smsall displacement theory (SDT), Sliding bearing, Roundness error, Oil film characteristic.

\section{Introduction}

As an important component of rotating machinery, sliding bearing rotor system has been widely used in rotating machinery fields such as large steam turbine, generator and engine camshaft system ${ }^{[1]}$. Due to the influence of machining accuracy of machine tools, manufacturing errors often exist on the machined bearing surface. Among them, roundness error is an important part of manufacturing error, and the roundness error of sliding bearing working surface and oil film thickness belong to the same order of magnitude $(\mu \mathrm{m})$, so the influence of roundness error on oil film characteristics of sliding bearing can not be ignored $^{[2]}$.

Scholars at home and abroad have made a series of researches on the influence of roundness error on oil film characteristics of sliding bearings. Radford ${ }^{[3]}$ used seven equidistant lobe journals to study the influence of journal roundness error on the performance of hydrodynamic sliding bearings, and compared the journal with and without

\footnotetext{
*Corresponding author: gxgxyjxxlb@163.com
} 
lobes through experiments. It was found that within a certain range of running speed and load, roundness error not only did no harm, but improved the lubrication boundary conditions of bearings to a certain extent, which was beneficial to the performance of bearings. Poznyak et $\mathrm{al}^{[4]}$ studied the influence of journal out-of-roundness on vibration characteristics of sliding bearing rotor system. The results show that the out-of-roundness of the shaft causes the double-frequency vibration of the bearing system. Xu et $\mathrm{al}^{[5]}$ carried out theoretical analysis and experimental research respectively. The research explored the influence of journal roundness error on the running characteristics of rotating machinery supported by sliding bearings. The results show that the out-of-roundness of journal directly affects the oil film thickness, and then affects the dynamic characteristics of the system. Zhang et $\mathrm{al}^{\left[{ }^{[6]}\right.}$ established the dynamic model of sliding bearing rotor system with elliptical error in the journal, and focused on the influence of elliptical error on the operating characteristics of bearing rotor system.

In this paper, SDT error modeling method is used to model the journal, and the influence of roundness error screw parameter on the running characteristics of sliding bearing is studied under the given journal roundness error, and the critical speed and stability of sliding bearing rotor system are emphatically analyzed.

\section{Mathematical model of roundness error based on SDT}

On any cross section of journal of sliding bearing, the intersection line (circle) between journal boundary and the cross section should be limited within the tolerance zone specified by roundness tolerance. As shown in fig 1, the journal SDT roundness error model uses vector equation to limit the intersection line between any cross section of the journal and its surface within the roundness tolerance zone with the tolerance value of $T_{\text {cir }}$. Therefore, the roundness error on the cylindrical surface can be described by vector equation (1):

$$
\left\{\begin{array}{l}
C_{d} \cdot\left(p-C_{p}\right)=0 \\
|| p-C_{p}\left|-r_{j}\right| \leq \frac{T_{C i r}}{2}
\end{array}\right.
$$

In which: $C_{d}$ is the orientation vector, $C_{p}$ is the positioning vector; $p$ is the coordinate vector of any point on the actual cylindrical surface.

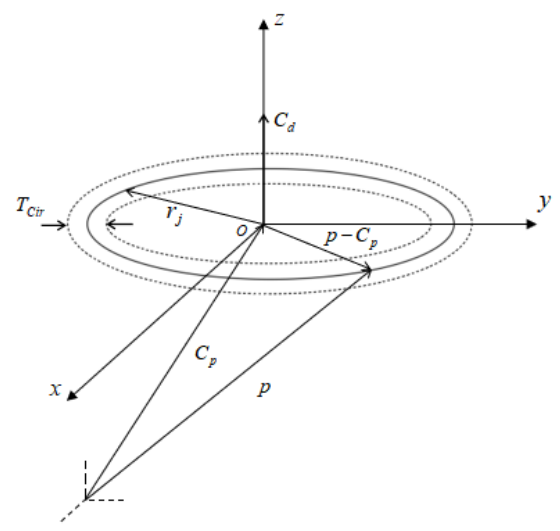

Fig. 1. Definition diagram of roundness error of journal.

The mathematical model of roundness error based on SDT can be expressed by formula $(2-2)^{[7]}$ : 


$$
-\frac{T_{\mathrm{Cir}}}{2} \leq \sqrt{\left(x+d_{x}\right)^{2}+\left(y+d_{y}\right)^{2}}-r_{j} \leq \frac{T_{\mathrm{Cir}}}{2}
$$

In which: $d x, d y$ are screw parameters representing roundness error, $r_{j}$ is the ideal journal radius, and $T_{\text {cir }}$ is the tolerance requirement of roundness error. All roundness error spinor parameters $\left(d x, d_{y}\right)$ conform to the variable inequality equation (3):

$$
-\frac{T_{\mathrm{Cir}}}{2} \leq d_{x} \leq \frac{T_{\mathrm{Cir}}}{2},-\frac{T_{\mathrm{Cir}}}{2} \leq d_{y} \leq \frac{T_{\mathrm{Cir}}}{2}
$$

\section{Dynamic model of sliding bearing rotor with roundness error and calculation of oil film thickness}

A dynamic model of sliding bearing rotor system is established, as shown in Fig 2. The influence of roundness error of journal surface on oil film characteristics of sliding bearing is studied by this model.

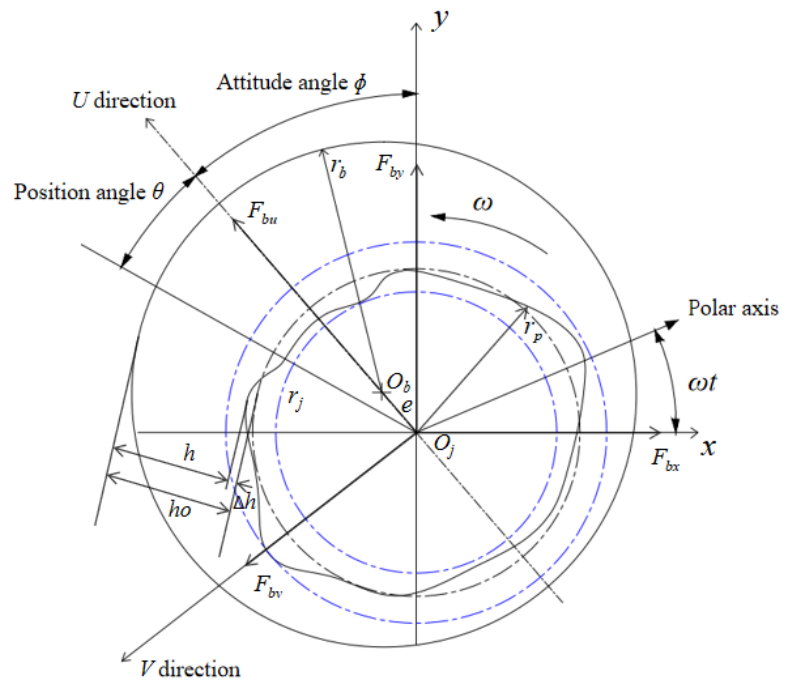

Fig. 2. Dynamic model of sliding bearing rotor system considering roundness error.

As shown in fig 2, a new generalized roundness error equation $r_{p}\left(\theta_{p}\right)$ can be derived based on SDT theory, which can be expressed by formula (4):

$$
\left\{\begin{array}{l}
r_{p}\left(\theta_{p}\right)=\sqrt{r_{j}^{2}+\Lambda^{2}-d_{x}^{2}-d_{y}^{2}}-\Lambda \\
\Lambda=\cos \theta_{p} \cdot d_{x}+\sin \theta_{p} \cdot d_{y} \\
\theta_{p}=\frac{\pi}{2}+\theta+\varphi-\omega t
\end{array}\right.
$$

Under the dynamic model of sliding bearing rotor system considering roundness error, the oil film thickness can be described by the ideal circular oil film thickness $h_{o}$ and the contour function $\Delta h$ which characterizes the surface roundness error.

The oil film thickness of the ideal round journal can be determined by formula (5):

$$
h_{0}=c(1+\varepsilon \cos \theta)
$$


In which: $r_{j}$ is the journal radius; $c$ is the bearing clearance; $\varepsilon$ is eccentricity, where $\varepsilon=e / c$.

According to SDT roundness error model, the oil film thickness of sliding bearing considering roundness error can be calculated, that is, the actual bearing oil film thickness function $h(\theta)$ can be expressed by formula (6):

$$
h(\theta)=c(1+\varepsilon \cos \theta)-r_{p}\left(\theta_{p}\right)+r_{j}
$$

\section{Calculation of oil film pressure and oil film force}

Sliding bearing rotor system works by changing oil film pressure. The nonlinear oil film force acting on the journal is related to both the position and the movement of the journal, so it can be obtained by numerical integration of the pressure field. When certain conditions are met, Reynolds equation under steady load can be simplified as shown in formula (7):

$$
\frac{\partial}{\partial x}\left(h^{3} \frac{\partial p}{\partial x}\right)+\frac{\partial}{\partial z}\left(h^{3} \frac{\partial p}{\partial z}\right)=6 \mu U_{t} \frac{\partial h}{\partial x}+12 \mu \frac{\partial h}{\partial t}
$$

In order to improve the solution accuracy, the Reynolds equation can be numerically solved by using the finite difference method under the Swift-Stieber boundary condition.

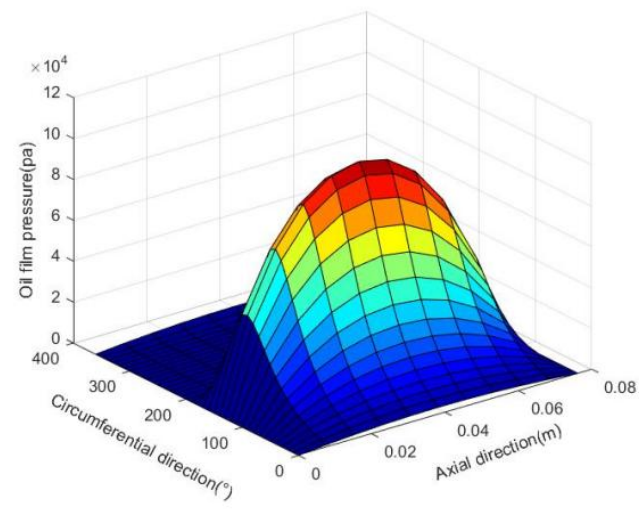

Fig. 3. Oil film pressure diagram.

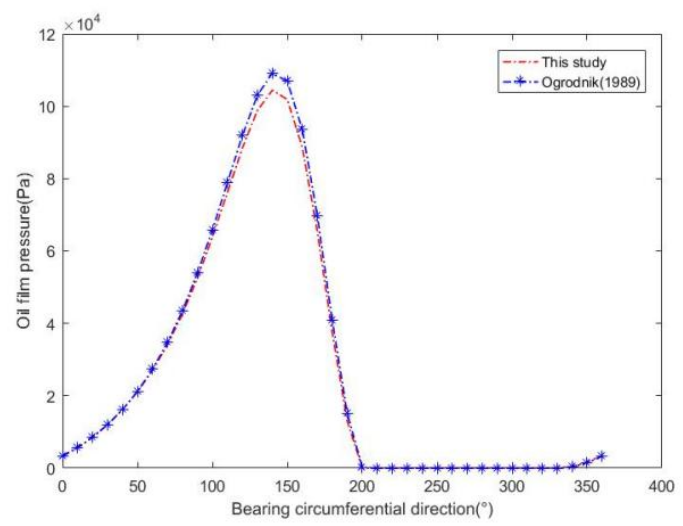

Fig. 4. Oil film pressure calibration chart. 
Fig 3 shows an example of oil film pressure distribution of ordinary sliding bearings. Fig 4 shows the comparison between the calculation example and the oil film pressure distribution results given by Ogrodnik ${ }^{[8]}$. As shown in the figure, the analysis results in this paper are consistent with the results in the literature, which shows the accuracy of the modeling method of shape and position error of sliding bearings based on SDT theory and can be used for subsequent research.

After the oil film pressure $p$ is obtained, the oil film pressure $p$ can be numerically integrated and then the oil film force components $F_{b u}$ and $F_{b v}$ in $U$ and $V$ directions can be calculated, as shown in formula (8):

$$
\left\{\begin{array}{l}
F_{b u}=\int_{0}^{m} \int_{1}^{n} p_{i, j} \sin \theta_{i, j} d x d z \\
F_{b v}=\int_{0}^{m} \int_{1}^{n} p_{i, j} \cos \theta_{i, j} d x d z
\end{array}\right.
$$

As shown in fig 2, the oil film forces $F_{b x}$ and $F_{b y}$ in the horizontal and vertical directions of the sliding bearing are obtained according to coordinate transformation, which can be expressed by formula (9):

$$
\left\{\begin{array}{l}
F_{b x}=-F_{b u} \sin \phi-F_{b v} \cos \phi \\
F_{b y}=F_{b u} \cos \phi-F_{b v} \sin \phi
\end{array}\right.
$$

\section{Influence of roundness error screw parameter on bearing oil film characteristics}

In the process of studying the influence of roundness error screw parameter on bearing oil film characteristics, the structural parameters of sliding bearing rotor system are shown in Table 1:

Table 1. Structure parameter table of sliding bearing rotor system.

\begin{tabular}{|c|c|}
\hline Parameter & Nominal dimension \\
\hline Bearing length- $L(\mathrm{~mm})$ & 76.2 \\
\hline Bearing diameter $-d_{b}(\mathrm{~mm})$ & 76.454 \\
\hline Journal diameter- $d_{j}(\mathrm{~mm})$ & 75.692 \\
\hline Draw ratio- $L / d_{b}$ & $1: 1$ \\
\hline Radius clearance- $c(\mathrm{~mm})$ & 0.381 \\
\hline Coefficient of viscosity $-\mu($ Pa.s $)$ & 0.017 \\
\hline
\end{tabular}

\subsection{Influence of roundness error on critical speed}

Studies at home and abroad show that the analysis of axis trajectory can be used as an important reference index to judge the stability of bearings. According to the convergence or divergence of the rotor axis trajectory, the critical speed of the sliding bearing rotor system can be determined. Next, the axis track of the rotor at different speeds is studied. When the initial eccentricity $\varepsilon=0.5$, the trend of the axis track of the rotor can be seen through observation, as shown in Fig 5. 


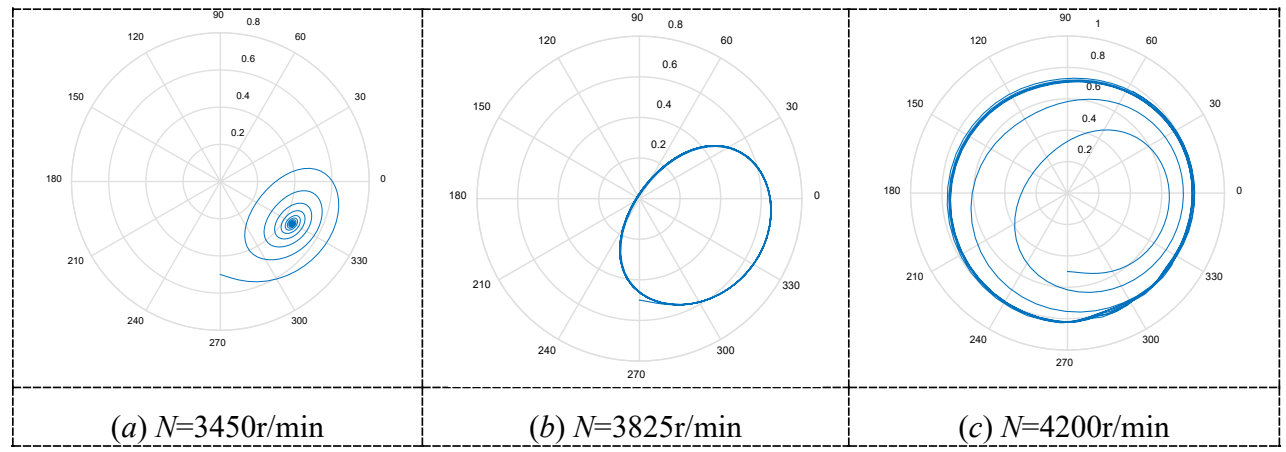

Fig. 5. Axis trajectories at critical speeds with different screw parameters.

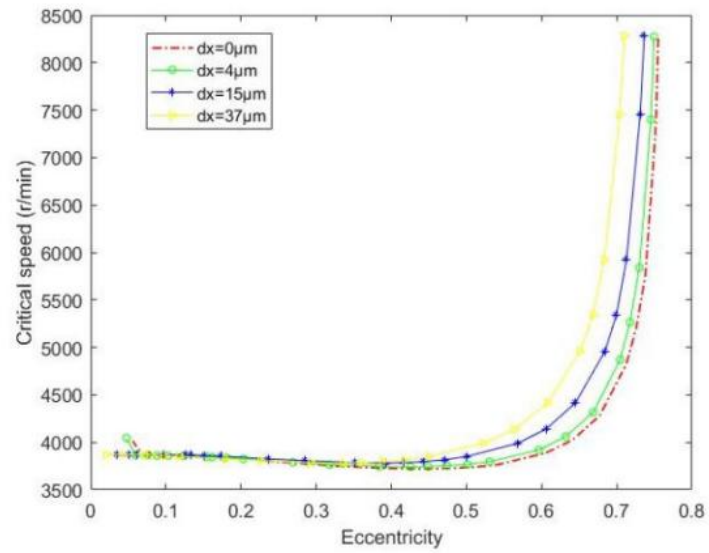

Fig. 6. Influence of roundness error on system stability.

As shown in fig $5(b)$, the rotor axis track is in a critical state at this speed, and the speed corresponding to this state is called the rotor critical speed, and the axis movement track of the shaft diameter is an elliptical ring. Figure 6 shows that the critical speed will increase with the increase of eccentricity, especially when eccentricity $\varepsilon>0.6$.

\subsection{Influence of roundness error on stability}

In order to better explain the influence of roundness error on the stability of bearing-rotor system, this paper introduces dimensionless stable operation parameter $O p$, and studies the influence of roundness error screw parameter on the stability of bearing-rotor system through the stability critical curve of Sommerfeld number and $O p$. The calculation formula (10) can be expressed as:

$$
O_{p}=\frac{F}{m c \omega^{2}}
$$

In which: $F$ is the bearing load; $m$ is the rotor mass; $\omega$ is the angular velocity of the rotor.

The relation curve between Sommerfeld number and dimension-stable operation parameter $O p$ is shown in fig 6 . When roundness error is small, the stability critical curve of sliding bearing with roundness error is very close to the critical curve of ideal circular sliding bearing system. At the same time, for the sliding bearing in this paper, the larger the 
roundness error value, the larger the stable area of the system, which improves the stability of the bearing rotor system. In addition, the results show that when Sommerfeld number $\mathrm{S}>0.6$, the roundness error has little influence on stability, and even can be ignored.

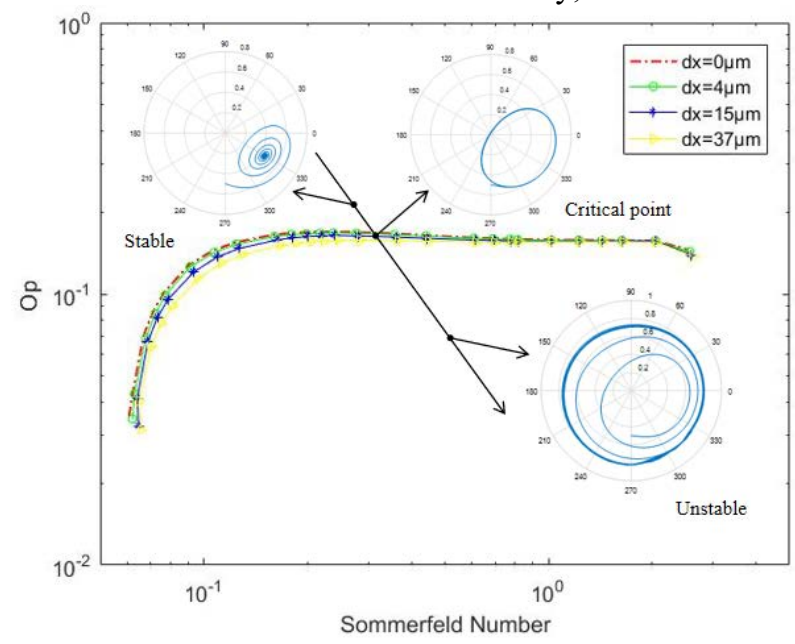

Fig. 7. Influence of roundness error on system stability.

\section{Conclusions}

(1) The existence of journal roundness error is beneficial to the stability of sliding bearing rotor system to a certain extent, and with the increase of roundness error screw parameter $\mathrm{dx}$, its promoting effect is more obvious. When Sommerfeld number $\mathrm{S}>0.6$, the roundness error of journal has little influence on stability.

(2) When the bearing-rotor system runs to a stable state at critical speed, the axis track is regular annular, and under the same screw parameters, the critical speed of the system will increase with the increase of eccentricity, and the increase of critical speed will be more obvious when $\varepsilon>0.6$.

This work was financially supported by the Guangxi Postgraduate Education Plan Innovation Project (YCSW2021318), Natural Science Foundation of Guangxi (2018GXNSFBA281195).

\section{References}

1. Bangchun Wen. Advanced Rotor Dynamics: Theory, Technology and Application[M]. Xian: Xian Jiaotong University Press, 2000: 5-20.

2. Haosheng Chen, Yongjian Li, darong Chen, et al. Numerical analysis of nonnewtonian fluid lubrication under regular morphology[J]. Journal of mechanical engineering, 2007(08): 48-52.

3. Radford A, Fitzgeorge D, The effects of journal lobbing on the performance of a hydrodynamic plain journal bearing[J]. Wear, 1977, 45(3): 311-322.

4. Poznyak E, Maiorov G. The calculation of rotor vibration caused by out-of-roundness of shafts in plain bearings[J]. Energomashinostroenie, 1981, 11: 7-11.

5. Wubin Xu, Ogrodnik P J, Li Bing, et al. Effect of journal out-of-roundness on stability of a symmetric hydrodynamic journal bearing system. Part 1: Theoretical analysis[J]. Proceedings of the Institution of Mechanical Engineers, 2015, 229(11); 1347-1358. 
6. ZhangYi lei, Wubin Xu, et al. Influence of ellipse error on the running characteristics of sliding bearing rotor system[J]. Mechanical Design and Manufacture, 2020(02): 240-243.

7. Min Cai. Research on mathematical modeling and analysis technology of tolerance of cylindrical elements based on mathematical definition[D]. Zhejiang University, 2002: 34-36.

8. Ogrodnik P J. Operation of rotor-bearing systems subjected to dynamic conical misalignment[D]. Staffordshire: Staffordshire University, 1989:36-39. 